\title{
A Note on the New Extended Beta Function with Its Application
}

\author{
Vandana Palsaniya $\mathbb{D},{ }^{1}$ Ekta Mittal $\mathbb{D},{ }^{2}$ Sunil Joshi $\mathbb{D},{ }^{3}$ and D. L. Suthar $\mathbb{D}^{4}$ \\ ${ }^{1}$ Department of Mathematics, St. Wilfred's PG. College, Jaipur, India \\ ${ }^{2}$ Department of Mathematics, IIS (Deemed to be University) Jaipur, Jaipur, India \\ ${ }^{3}$ Department of Mathematics and Statistics, Manipal University Jaipur, Jaipur, Rajasthan, India \\ ${ }^{4}$ Department of Mathematics, Wollo University, P.O. Box: 1145, Dessie, Ethiopia \\ Correspondence should be addressed to D. L. Suthar; dlsuthar@gmail.com
}

Received 4 October 2021; Revised 9 November 2021; Accepted 18 November 2021; Published 16 December 2021

Academic Editor: Nasser Saad

Copyright (c) 2021 Vandana Palsaniya et al. This is an open access article distributed under the Creative Commons Attribution License, which permits unrestricted use, distribution, and reproduction in any medium, provided the original work is properly cited.

\begin{abstract}
The purpose of this research is to provide a systematic review of a new type of extended beta function and hypergeometric function using a confluent hypergeometric function, as well as to examine various belongings and formulas of the new type of extended beta function, such as integral representations, derivative formulas, transformation formulas, and summation formulas. In addition, we also investigate extended Riemann-Liouville (R-L) fractional integral operator with associated properties. Furthermore, we develop new beta distribution and present graphically the relation between moment generating function and $\ell$.
\end{abstract}

\section{Introduction}

Special functions, often denoted by series, emerge as a result of solving various problems in classical physics. Typically, these problems demand the flow of electromagnetic, acoustic, or thermal energy. It has developed into a vital resource for the particular and specialized roles played by scientists and engineers these days. These characteristics are critical in a variety of domains, including physical science, mathematics, and engineering. The Gaussian hypergeometric function ${ }_{2} F_{1}$ is among most important special functions. The hypergeometric function ${ }_{2} F_{1}$ and its numerous generalizations have been investigated by many researchers who have provided a notably large number of their formula properties (see, e.g., [1-7]). Over the last decades, many researchers have established the generalizations of the classical gamma and beta functions and provided a number of intriguing and useful properties for the extended functions (see, e.g., [8-16]).

We begin with recalling some definitions of extended beta functions.

Chaudhary and Zubair [2] extended the classical gamma function as follows:

$$
\Gamma_{\varrho}(x)=\int_{0}^{\infty} \ell^{x-1} e^{(-\ell-(\varrho / \ell))} \mathrm{d} \ell,
$$

where $\{\mathfrak{R e}(\varrho)>0, \mathfrak{R e}(x)>0\}$.

Subsequently, in 1997, Chaudhary et al. [17] developed the beta function extension

$$
B_{\mathrm{Q}}(x, y)=\int_{0}^{1} \ell^{x-1}(1-\ell)^{y-1} e^{(-\rho / \ell(1-\ell))} \mathrm{d} \ell,
$$

where $\{\mathfrak{R e}(\varrho)>0, \mathfrak{R} \mathfrak{e}(x)>0, \mathfrak{R e}(y)>0\}$.

Further, Chaudhary et al. [3] used the extended beta function (2) to present the following extension of the hypergeometric function:

$$
F_{\varrho}(\dot{a}, \dot{b} ; \dot{c} ; \vartheta)=\sum_{n=0}^{\infty} \frac{(a)_{n} B_{\varrho}(b+n, \bar{c}-b)}{\mathfrak{B}(b, \hat{c}-b)} \frac{\vartheta^{n}}{n !},
$$

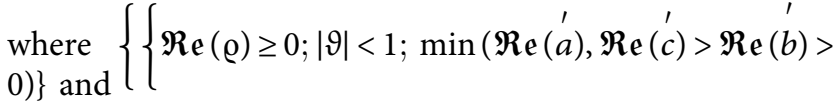

$$
\varphi_{\mathrm{\varrho}}(b ; c ; \vartheta)=\sum_{n=0}^{\infty} \frac{B_{\mathrm{e}}(b+n, c-b)}{\mathfrak{B}(b, c-b)} \frac{\vartheta^{n}}{n !},
$$


where $\left\{\mathfrak{R e}(\varrho) \geq 0 ;|\vartheta|<1 ; \mathfrak{R e}{ }^{\prime}(c)>\operatorname{Re}(\dot{b})>0\right\}$.

The Pochhammer symbol $(\sigma)_{n}$ is defined (for $\sigma, n \in C$ ), in terms of the familiar gamma function $\Gamma$, by (see, e.g., [17], p. 2 and p. 5)

$$
\begin{aligned}
(\sigma)_{n} & :=\frac{\Gamma(\sigma+n)}{\Gamma(\sigma)},(\sigma+n) \in C \backslash Z_{0}^{-} \\
& =\left\{\begin{array}{cc}
1 & n=0 \\
\sigma(\sigma+1) \ldots(\sigma+n-1) & n \in N
\end{array}\right\} .
\end{aligned}
$$

Here, let $C, Z_{0}^{-}$, and $N$ denote the sets of complex numbers, nonpositive integers, and positive integers, respectively.

Ozergin et al. [11] described the generalization of the beta function such as

$$
B_{\varrho}^{(\sigma, \delta)}(x, y)=\int_{0}^{1} \ell^{x-1}(1-\ell)^{y-1}{ }_{1} F_{1}\left(\sigma ; \delta ; \frac{-\varrho}{\ell(1-\ell)}\right) \mathrm{d} \ell .
$$

Chand et al. [18] recently described the latest extension of the beta function as follows:

$$
B_{\kappa}^{\mathrm{MC}}(x, y)=\int_{0}^{1} \ell^{x-1}(1-\ell)^{y-1} e^{\kappa \ell(1-\ell)} \mathrm{d} \ell
$$

where $\{\mathfrak{R e}(x)>0, \mathfrak{R e}(y)>0, \kappa \in C ;|\kappa|<K\}$ and $\left\{K \in R^{+}\right.$ and not greater than 2.0335$\}$.

In this paper, we aim to introduce and investigate extended beta function, extended hypergeometric function, and extended confluent hypergeometric function. To do this, we have divided the paper into three portions. The first part discusses the introductory segment that has been documented by many researchers. In the second part, we gain Mellin transformations, summation relationships, beta distributions, integral representations of hypergeometric and confluent hypergeometric functions, differentiation formulas, and transformation formulas incorporating this new extended beta function. In the last part, we develop a novel property-rich extension of the fractional derivative Riemann-Liouville operator.

\section{New Extended Beta Function and Its Properties}

In this segment, we propose new generalization of classical beta function as follows:

$$
B_{\sigma, \delta ; \kappa}^{\mathrm{MC}}(x, y)=\int_{0}^{1} \ell^{x-1}(1-\ell)^{y-1}{ }_{1} F_{1}(\sigma ; \delta ; \kappa \ell(1-\ell)) \mathrm{d} \ell,
$$

where $\left\{\mathfrak{R e}(\sigma)>0, \mathfrak{R e}(\delta)>0, \kappa \in C,|\kappa|<K ; K \in R^{+}\right.$and less than 2.0335 .

If $\kappa=0$ and $\sigma=\delta$, then equation (8) reduces to classical beta function.

\subsection{Main Result}

2.1.1. Melline Transform Representation of the New Generalisation of Beta Function

Theorem 1. If $\mathfrak{R e}(x-\tau)>0, \mathfrak{R e} \quad(y-\tau)>0, \mathfrak{R e}(\sigma)>$ $\mathfrak{R e}(\delta)>\mathfrak{R e}(\tau)>0, \kappa \in C$ and $|\kappa|<K ; K \in R^{+}$and less than 2.0335 , then

$$
\int_{0}^{\infty} \kappa^{\tau-1} B_{\sigma, \delta ; \kappa}^{\mathrm{MC}}(x, y) \mathrm{d} \kappa=(-1)^{\tau}\left(\frac{\Gamma(\tau) \Gamma(\delta) \Gamma(\sigma-\delta)}{\Gamma(\sigma) \Gamma(\delta-\tau)}\right) B(x-\tau, y-\tau)
$$

Proof. Taking the left hand side and using equation (8), we have

$$
\int_{0}^{\infty} \kappa^{\tau-1} B_{\sigma, \delta ; \kappa}^{\mathrm{MC}}(x, y) \mathrm{d} \kappa=\int_{0}^{\infty} \kappa^{\tau-1}\left\{\int_{0}^{1} \ell^{x-1}(1-\ell)^{y-1}{ }_{1} F_{1}(\sigma ; \delta ; \kappa \ell(1-\ell)) \mathrm{d} \ell\right\} \mathrm{d} \kappa
$$

Now, interchanging the order of integration of equation (10) and forming uniform convergence, we have 


$$
=\int_{0}^{1} \ell^{x-1}(1-\ell)^{y-1}\left\{\int_{0}^{\infty} \kappa^{\tau-1}{ }_{1} F_{1}(\sigma ; \delta ; \kappa \ell(1-\ell)) \mathrm{d} \kappa\right\} \mathrm{d} \ell .
$$

Substituting $\kappa \ell(1-\ell)=-\varsigma$ in the above expression, we get

$$
\begin{aligned}
& =\int_{0}^{1} \ell^{x-1}(1-\ell)^{y-1} \int_{0}^{\infty}\left(\frac{-\varsigma}{\ell(1-\ell)}\right)^{\tau-1}{ }_{1} F_{1}(\sigma ; \delta ;-\varsigma) \frac{-\mathrm{d} \varsigma}{\ell(1-\ell)} \mathrm{d} \ell \\
& =\int_{0}^{1} \ell^{x-\tau-1}(1-\ell)^{y-\tau-1}(-1)^{\tau} \int_{0}^{\infty}(\varsigma)^{\tau-1}{ }_{1} F_{1}(\sigma ; \delta ;-\varsigma) \mathrm{d} \varsigma \mathrm{d} \ell .
\end{aligned}
$$

Using result (page no. 821, [5]), we obtain

$$
=(-1)^{\tau}\left(\frac{\Gamma(\tau) \Gamma(\delta) \Gamma(\sigma-\delta)}{\Gamma(\sigma) \Gamma(\delta-\tau)}\right) B(x-\tau, y-\tau) .
$$

Theorem 2. For the new generalized beta function, we have the subsequent integral representation of new classical beta function. If $\mathfrak{R e}(x)>0, \mathfrak{R e}(y)>0, \mathfrak{R e}(\sigma)>\operatorname{Re}(\delta)>0, \kappa \in C$, and $|\kappa|<K ; K \in R^{+}$and less than 2.0335, then

$$
\begin{aligned}
& B_{\sigma, \delta ; \kappa}^{\mathrm{MC}}(x, y)=2 \int_{0}^{(\pi / 2)} \cos ^{2 x-1} \theta \sin ^{2 y-1} \theta_{1} F_{1}\left(\sigma ; \delta ; \kappa \cos ^{2} \theta \sin ^{2} \theta\right) \mathrm{d} \theta, \\
& B_{\sigma, \delta ; \kappa}^{\mathrm{MC}}(x, y)=\int_{0}^{\infty} \frac{\varsigma^{x-1}}{(1+\varsigma)^{x+y}} F_{1}\left(\sigma ; \delta ; \frac{\kappa \varsigma}{(1+\varsigma)^{2}}\right) \mathrm{d} \varsigma,
\end{aligned}
$$

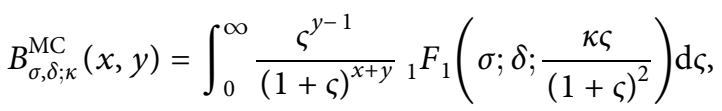

$$
\begin{aligned}
& B_{\sigma, \delta ; \kappa}^{\mathrm{MC}}(x, y)=\frac{1}{2} \int_{0}^{\infty} \frac{\varsigma^{x-1}+\varsigma^{y-1}}{(1+\varsigma)^{x+y}} F_{1}\left(\sigma ; \delta ; \frac{\kappa \varsigma}{(1+\varsigma)^{2}}\right) \mathrm{d} \varsigma,
\end{aligned}
$$

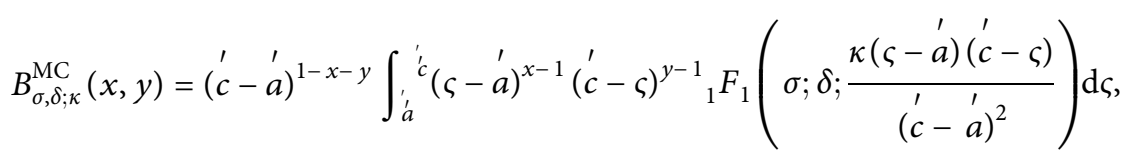

$$
\begin{aligned}
& B_{\sigma, \delta ; \kappa}^{\mathrm{MC}}(x, y)=2^{1-x-y} \int_{-1}^{1}(1+\varsigma)^{x-1}(1-\varsigma)^{y-1}{ }_{1} F_{1}\left(\sigma ; \delta ; \frac{\kappa\left(1-\varsigma^{2}\right)}{4}\right) \mathrm{d} \varsigma, \\
& B_{\sigma, \delta ; \kappa}^{\mathrm{MC}}(x, y)=2^{1-x-y} \int_{-\infty}^{\infty} e^{(x-y) \ell}{ }_{1} F_{1}\left(\sigma ; \delta ; \frac{\kappa}{4 \cosh ^{2} \ell}\right) \frac{1}{(\cosh \ell)^{x+y}} \mathrm{~d} \ell .
\end{aligned}
$$

Proof: Equations (14) and (15) can be easily obtained by substituting $\ell=\cos ^{2} \theta$ and $\ell=(\varsigma /(1+\varsigma))$ in equation (8). Equation (16) can be obtained by interchanging $x$ and $y$; equation (17) can be obtained by adding equations (15) and (16) with some simplifications. Equation (18) can be achieved by putting $\ell=((\varsigma-a) /(c-a))$ in equation (8), and replacing $a=-1, c=1$ in equation (18), we get equation (19) and finally for the result given in equation (20), it can be obtained by putting $\varsigma=\tanh (\ell)$ and using $\tanh (\ell)=\left(\left(e^{\ell}-\right.\right.$ $\left.\left.e^{-\ell}\right) /\left(e^{\ell}+e^{-\ell}\right)\right)$ in equation (19).
Throughout the paper, we consider the condition $\mathfrak{R e}(\sigma)>\mathfrak{R e}(\delta)>0, \kappa \in C$, and $|\kappa|<K ; K \in R^{+}$and less than 2.0335 .

\subsubsection{Summation Relation}

Theorem 3. The new generalized beta function: if $\mathfrak{R e}(x)>0, \mathfrak{R e}(y)>0$, then we have the subsequent functional relation: 


$$
B_{\sigma, \delta ; \kappa}^{\mathrm{MC}}(x, y+1)+B_{\sigma, \delta ; \kappa}^{\mathrm{MC}}(x+1, y)=B_{\sigma, \delta ; \kappa}^{\mathrm{MC}}(x, y) .
$$

Proof: Taking the left hand side of equation (21), we get

$$
\begin{aligned}
& B_{\sigma, \delta ; \kappa}^{\mathrm{MC}}(x, y+1)+B_{\sigma, \delta ; \kappa}^{\mathrm{MC}}(x+1, y) \\
& =\int_{0}^{1} \ell^{x-1}(1-\ell)^{y+1-1}{ }_{1} F_{1}(\sigma ; \delta ; \kappa \ell(1-\ell)) \mathrm{d} \ell \\
& \quad+\int_{0}^{1} \ell^{x+1-1}(1-\ell)^{y-1}{ }_{1} F_{1}(\sigma ; \delta ; \kappa \ell(1-\ell)) \mathrm{d} \ell \\
& =\int_{0}^{1}\left[\ell^{x-1}(1-\ell)^{y}+\ell^{x}(1-\ell)^{y-1}\right]_{1} F_{1}(\sigma ; \delta ; \kappa \ell(1-\ell)) \mathrm{d} \ell \\
& =\int_{0}^{1} \ell^{x-1}(1-\ell)^{y-1}{ }_{1} F_{1}(\sigma ; \delta ; \kappa \ell(1-\ell)) \mathrm{d} \ell,
\end{aligned}
$$

which is a required result.

Remark 1. If we choose $\kappa=0$ and $\sigma=\delta$, in the above equation (21), then it will convert into the classical beta function.

Theorem 4. If $\mathfrak{R e}(x)>0, \mathfrak{R e}(y)>0$, then we have the subsequent summation relation:

$$
B_{\sigma, \delta ; \kappa}^{\mathrm{MC}}(x, 1-y)=\sum_{n=0}^{\infty} \frac{(y)_{n}}{n !} B_{\sigma, \delta ; \kappa}^{\mathrm{MC}}(x+n, 1) .
$$

Proof. We start with the left hand side of equation (23) with the help of equation (8),

$$
\begin{aligned}
B_{\sigma, \delta ; \kappa}^{\mathrm{MC}}(x, 1-y) & =\int_{0}^{1} \ell^{x-1}(1-\ell)^{1-y-1}{ }_{1} F_{1}(\sigma ; \delta ; \kappa \ell(1-\ell)) \mathrm{d} \ell \\
& =\int_{0}^{1} \ell^{x-1}(1-\ell)^{-y}{ }_{1} F_{1}(\sigma ; \delta ; \kappa \ell(1-\ell)) \mathrm{d} \ell .
\end{aligned}
$$

Using the binomial expansion $(1-\ell)^{-y}=\sum_{n=0}^{\infty}$ $\left((y)_{n} \ell^{n} / n !\right),|\ell|<1$ in the above expression, and switching the order of summation and integration, we attain

$$
\begin{aligned}
B_{\sigma, \delta ; \kappa}^{\mathrm{MC}}(x, 1-y) & =\sum_{n=0}^{\infty} \frac{(y)_{n}}{n !} \int_{0}^{1} \ell^{x+n-1}{ }_{1} F_{1}(\sigma ; \delta ; \kappa \ell(1-\ell)) \mathrm{d} \ell \\
& =\sum_{n=0}^{\infty} \frac{(y)_{n}}{n !} B_{\sigma, \delta ; \kappa}^{\mathrm{MC}}(x+n, 1) .
\end{aligned}
$$

Theorem 5. If $\mathfrak{R e}(x)>0, \mathfrak{R e}(y)>0$, we have the subsequent summation relation:

$$
B_{\sigma, \delta ; \kappa}^{\mathrm{MC}}(x, y)=\sum_{n=0}^{\infty} B_{\sigma, \delta ; \kappa}^{\mathrm{MC}}(x+n, 1+y)
$$

Proof: Considering the binomial series expansion,

$$
\begin{aligned}
(1-\ell)^{y-1} & =(1-\ell)^{y}(1-\ell)^{-1} \\
& =(1-\ell)^{y} \sum_{n=0}^{\infty} \ell^{n}, \quad|\ell|<1,
\end{aligned}
$$

using the above result in equation (8) and interchanging the summation and integration, then we have

$$
\begin{aligned}
B_{\sigma, \delta ; \kappa}^{\mathrm{MC}}(x, y) & =\int_{0}^{1} \ell^{x+n-1}(1-\ell)^{y+1-1}{ }_{1} F_{1}(\sigma ; \delta ; \kappa \ell(1-\ell)) \mathrm{d} \ell \\
& =\sum_{n=0}^{\infty} B_{\sigma, \delta ; \kappa}^{M C}(x+n, y+1) .
\end{aligned}
$$

2.1.3. The Beta Distribution of Extended Beta Function $B_{\sigma, \delta ; \kappa}^{M C}(x, y)$. The specification of the new generalized beta function is the beta distribution in statistics. We are now describing new generalized beta distributions

$$
f(\ell)=\left\{\frac{1}{B_{\sigma, \delta ; \kappa}^{\mathrm{MC}}(x, y)} \ell^{x-1}(1-\ell)^{y-1}{ }_{1} F_{1}(\sigma ; \delta ; \kappa \ell(1-\ell) 0<\ell<10 \text { therwise }\} .\right.
$$

The generalized beta distribution is with parameters $x$ and $y$ such that $-\infty<x<\infty,-\infty<y<\infty$ and $\mathfrak{R e}(\sigma)>0, \mathfrak{R e}(\delta)>0,|\kappa|<K ; K \in \mathfrak{R}^{+}$and less than 2.0335 will be applied to a random variable $X$ with a probability density function (pdf) assumed by $f(\ell)$.

If $v$ is any real number, then

$$
\mathfrak{E}\left(X^{v}\right)=\frac{B_{\sigma, \delta ; \kappa}^{\mathrm{MC}}(x+v, y)}{B_{\sigma, \delta ; \kappa}^{\mathrm{MC}}(x, y)}
$$

and for $v=1$, mean of the distribution is

$$
\mu=\mathfrak{F}(X)=\frac{B_{\sigma, \delta ; \kappa}^{\mathrm{MC}}(x+1, y)}{B_{\sigma, \delta ; \kappa}^{\mathrm{MC}}(x, y)},
$$


and variance of the distribution is

$$
\begin{aligned}
\sigma^{2} & =\mathfrak{E}\left(X^{2}\right)-\mathfrak{F}(X)^{2} \\
& =\frac{B_{\sigma, \delta ; \kappa}^{\mathrm{MC}}(x+2, y)}{B_{\sigma, \delta: \kappa}^{\mathrm{MC}}(x, y)}-\left\{\frac{B_{\sigma, \delta: \kappa}^{\mathrm{MC}}(x+1, y)}{B_{\sigma, \delta ; \kappa}^{\mathrm{MC}}(x, y)}\right\}^{2} \\
& =\frac{B_{\sigma, \delta ; \kappa}^{\mathrm{MC}}(x, y) B_{\sigma, \delta ; \kappa}^{\mathrm{MC}}(x+2, y)-\left\{B_{\sigma, \delta ; \kappa}^{\mathrm{MC}}(x+1, y)\right\}^{2}}{\left\{B_{\sigma, \delta ; \kappa}^{\mathrm{MC}}(x, y)\right\}^{2}} .
\end{aligned}
$$

The moment generating function of the distribution is

$$
\begin{aligned}
\mathfrak{M}_{x}(\ell) & =\sum_{n=0}^{\infty} \frac{\ell^{n}}{n !} \mathfrak{E}\left(X^{n}\right) \\
& =\frac{1}{B_{\sigma, \delta: \kappa}^{\mathrm{MC}}(x, y)} \sum_{n=0}^{\infty} B_{\sigma, \delta: \kappa}^{M C}(x+n, y) \frac{\ell^{n}}{n !},
\end{aligned}
$$

and cumulative frequency distribution of $f(\ell)$ can be expressed as

$$
\mathfrak{F}(x)=\frac{B_{\sigma, \delta: \kappa, \vartheta}^{\mathrm{MC}}(x, y)}{B_{\sigma, \delta: \kappa}^{\mathrm{MC}}(x, y)},
$$

where

$$
\begin{aligned}
B_{\sigma, \delta ; \kappa, \vartheta}^{\mathrm{MC}}(x, y)= & \int_{0}^{\vartheta} \ell^{x-1}(1-\ell)^{y-1}{ }_{1} F_{1}(\sigma ; \delta ; \kappa \ell(1-\ell)) \mathrm{d} \ell, \\
& \left\{\mathfrak{R e}(\sigma)>0, \mathfrak{R e}(\delta)>0, \kappa \in C,|\kappa|<K ; K \in \mathfrak{R}^{+},-\infty<x<\infty,-\infty<y<\infty\right\},
\end{aligned}
$$

which is the new extended incomplete beta function, for $\kappa=0, x, y>0$, equation (35) convergence, and
$B_{\sigma, \delta ; 0, \vartheta}^{\mathrm{MC}}(x, y)=B_{\vartheta}(x, y)$, where $B_{\vartheta}(x, y)$ is the incomplete beta function (page no. 960, [5]) demarcated by

$$
\int_{0}^{\vartheta} \ell^{x-1}(1-\ell)^{y-1} \mathrm{~d} \ell=B_{\vartheta}(x, y)=B_{\sigma, \delta ; 0, \vartheta}^{\mathrm{MC}}(x, y)=\frac{\vartheta^{x}}{x^{2}} F_{1}(x ; 1-y ; x+1 ; \vartheta) .
$$

It is also given by the series

$$
=\vartheta^{x} \sum_{n=0}^{\infty} \frac{(1-y)_{n} \vartheta^{n}}{n !(x+n)}
$$

where $(1-y)_{n}$ is the Pochhammer symbol. It is to be noted that the problem of stating $B_{\sigma, \delta ; \kappa, \vartheta}^{\mathrm{MC}}(x, y)$ in the form of other special functions leftovers open. Apparently, this distribution should be convenient in extending the statistical results for variables that are strictly positive to deal with those variables that can take large negative values arbitrarily.

2.2. Graphical Representation between $\mathfrak{M}_{x}$ and $\ell$. Figure 1 describes the graphical form of equation (33) by using different values of $\kappa$ within the range $(-2,2)$ and some fixed values of $x, y, \sigma, \delta$. We can see that the graph is decreasing in nature. The different values between $\mathfrak{M}_{x}(\ell)$ and $\ell, \kappa$ are given in Table 1 .

2.2.1. Generalised Gauss Hypergeometric and Confluent Hypergeometric Function. Here, we define the generalized hypergeometric function and confluent hypergeometric function with the help of new generalization of classical beta function given in equation (8).

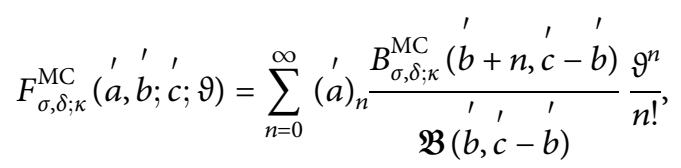

$$
\varphi_{\sigma, \delta ; \kappa}^{\mathrm{MC}}(b ; c ; \vartheta)=\sum_{n=0}^{\infty} \frac{B_{\sigma, \delta ; \kappa}^{\mathrm{MC}}(\dot{b}+n, c-b)}{\mathfrak{B}(b, c-\dot{b})} \frac{\vartheta^{n}}{n !}
$$

where $\left\{|\vartheta|<1, \operatorname{Re}(\stackrel{a}{a})>0, \operatorname{Re}\left({ }^{\prime}\right)>\operatorname{Re}(b)>0\right\}$, then

$$
\begin{aligned}
& F_{\sigma, \sigma ; \kappa}^{\mathrm{MC}}\left(\begin{array}{l}
\prime \\
(a, b ; c ; \vartheta
\end{array}\right)=F_{\kappa}^{\mathrm{MC}}\left(\begin{array}{l}
\prime \\
(a, b ; c ; \vartheta),
\end{array}\right. \\
& F_{\sigma, \sigma ; 0}^{\mathrm{MC}}(\dot{a}, \dot{b} ; \dot{c} ; \vartheta)={ }_{2} F_{1}(\dot{a}, \dot{b} ; \dot{c} ; \vartheta), \\
& \varphi_{\sigma, \sigma ; \kappa}^{\mathrm{MC}}\left(\begin{array}{l}
\prime \\
b ; c ; \vartheta)
\end{array}\right) \varphi_{\kappa}^{\mathrm{MC}}\left(\begin{array}{l}
\prime \\
(b ; c ; \vartheta
\end{array}\right. \\
& \varphi_{\sigma, \sigma ; 0}^{\mathrm{MC}}\left(\begin{array}{l}
\prime \\
b ; c ; \vartheta
\end{array}\right)={ }_{1} \varphi_{1}(b ; c ; \vartheta) .
\end{aligned}
$$

2.2.2. Integral Representation of Gauss and Confluent Hypergeometric Function by Using New Generalization of Beta Function

Theorem 6. If $|\arg (1-\vartheta)|<\pi, \quad \mathfrak{R e}(a)>0, \mathfrak{R e}(c)>$ $\mathfrak{R e}(b)>0$, then fon the new generalized Gauss hypergeometric function $F_{\sigma, \delta ; \kappa}^{M C}(a, b ; c ; \vartheta)$, we have the subsequent integral representation demarcated as 


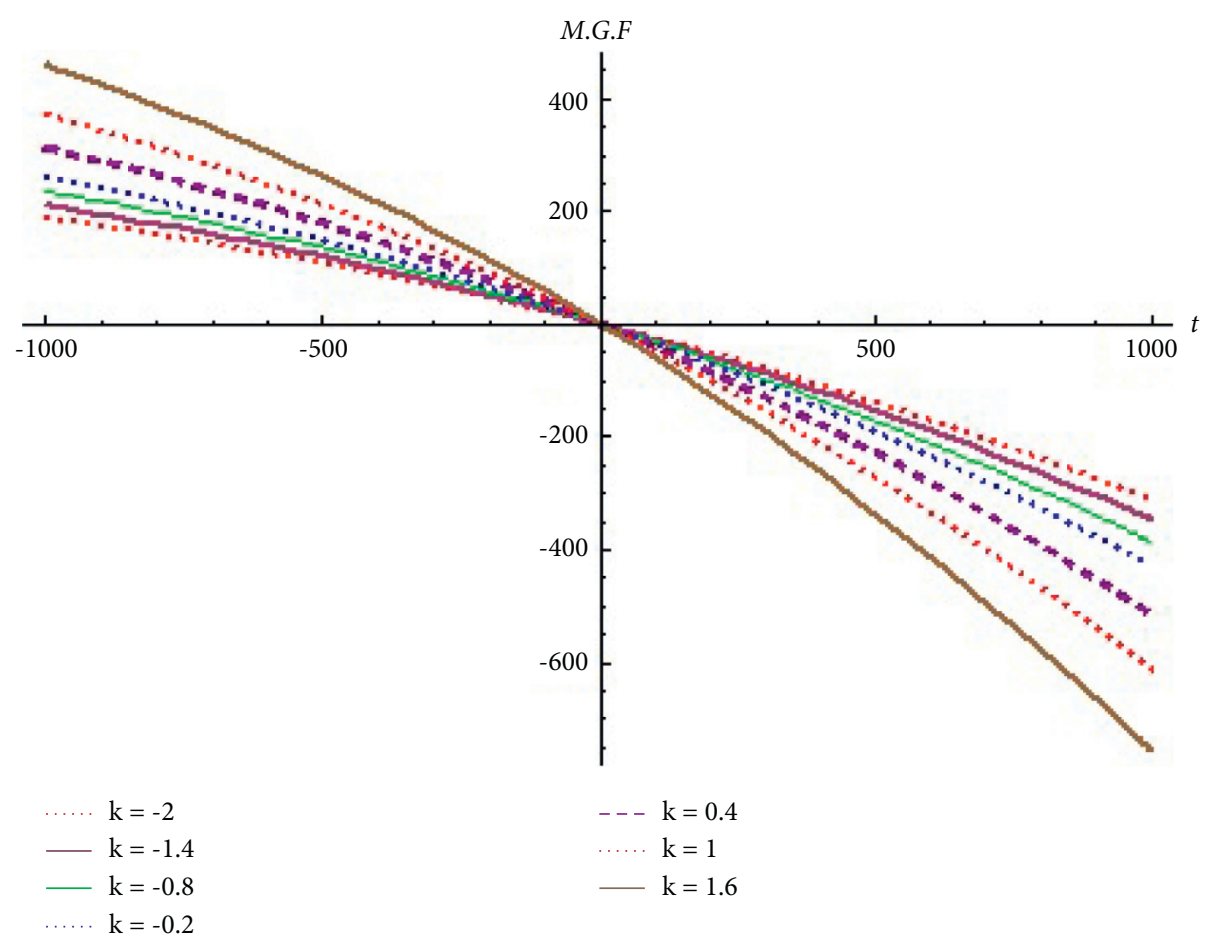

Figure 1: Curve between $\ell$ and M.G.F with different value of $\kappa$ with different values with some suitable assumption $x=4, y=5, \sigma=2, \delta=3 ;|\kappa|<2.0335$.

TABLE 1: Tabular representation of $\mathfrak{M}_{x}(\ell)$ and $\ell, \kappa$.

\begin{tabular}{|c|c|c|c|c|c|c|c|}
\hline \multirow{2}{*}{$\begin{array}{l}\ell \downarrow \\
\kappa \longrightarrow \\
\end{array}$} & \multicolumn{7}{|c|}{$\mathfrak{M}_{x}(\ell)$} \\
\hline & -2 & -1.4 & -0.8 & -0.2 & 0.4 & 1.0 & 1.6 \\
\hline-1000 & 190.189 & 210.91 & 236.212 & 261.006 & 313.452 & 372.309 & 460.187 \\
\hline-700 & 144.256 & 159.945 & 179.103 & 197.876 & 237.586 & 282.15 & 348.687 \\
\hline-400 & 89.4357 & 99.1201 & 110.946 & 122.534 & 147.046 & 174.555 & 215.627 \\
\hline-100 & 24.8554 & 27.4676 & 30.6573 & 33.783 & 40.3948 & 47.8147 & 58.8931 \\
\hline 200 & -50.4204 & -56.0507 & -62.9257 & -69.6627 & -83.9136 & -99.9063 & -123.784 \\
\hline 500 & -137.396 & -152.548 & -171.061 & -189.182 & -227.536 & -270.677 & -334.84 \\
\hline 800 & -237.147 & -263.219 & -295.057 & -326.254 & -326.254 & -466.309 & -576.884 \\
\hline
\end{tabular}

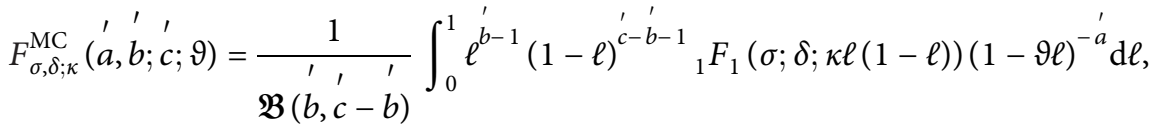

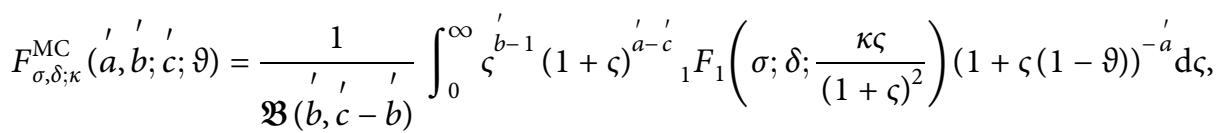

$$
\begin{aligned}
& F_{\sigma, \delta ; \kappa}^{\mathrm{MC}}(a, b ; c ; \vartheta)=\frac{2}{\mathfrak{B}(b, c-b)} \int_{0}^{(\pi / 2)} \sin ^{2 b-1} \theta \cos ^{2 c-2 b-1} \theta\left(1-\vartheta \sin ^{2} \theta\right)^{-{ }^{\prime}}{ }_{1}^{\prime} F_{1}\left(\sigma ; \delta ; \kappa \sin ^{2} \theta \cos ^{2} \theta\right) \mathrm{d} \theta
\end{aligned}
$$

Proof: Considering the left hand side of equation (41) by means of equations (38) and (8), we yield 


$$
F_{\sigma, \delta ; \kappa}^{\mathrm{MC}}\left(a, b^{\prime} ; c^{\prime} ; \vartheta\right)=\frac{1}{\mathfrak{B}(b, c-b)} \sum_{n=0}^{\infty}(a)_{n} \int_{0}^{1} \ell^{\prime} e^{b+n-1}(1-\ell)^{\prime c-b-1}{ }_{1}^{\prime} F_{1}(\sigma ; \delta ; \kappa \ell(1-\ell)) \frac{\vartheta^{n}}{n !} \mathrm{d} \ell .
$$

Changing the order of integration and summation with some simplifications, we attain the right hand side of Further, putting $\varsigma=(\ell /(1-\ell))$ in $(41)$, we get equation (41).

$$
=\frac{1}{\mathfrak{B}(b, c-b)} \int_{0}^{\infty} \varsigma^{b^{b-1}}(1+\varsigma)^{\mathfrak{a}^{\prime}-{ }_{1}} F_{1}\left(\sigma ; \delta ; \frac{\kappa \varsigma}{(1+\varsigma)^{2}}\right)(1+\varsigma(1-9))^{-{ }^{\prime}} \mathrm{d} \varsigma
$$

Again, if we choose $\ell=\sin ^{2} \theta$ in equation (41), we have

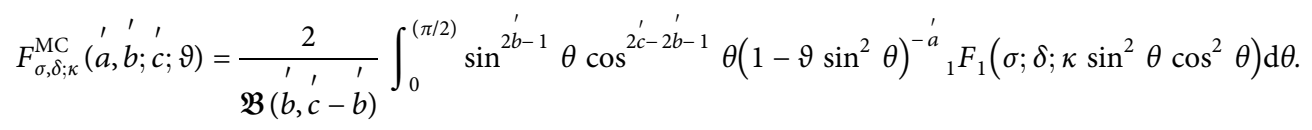

Remark 2. By choosing $9=1$ in equation (41), we have the subsequent relation between new generalization of Gauss hypergeometric function

$$
\begin{aligned}
& F_{\sigma, \delta ; \kappa}^{\mathrm{MC}}\left({ }^{\prime}, b^{\prime} ; c ; 1\right)=\frac{1}{\mathfrak{B}(b, c-b)} \int_{0}^{1} \ell^{b^{b-1}}(1-\ell)^{c^{c}-a^{\prime}-b_{-1}}{ }_{1} F_{1}(\sigma ; \delta ; \kappa \ell(1-\ell)) \mathrm{d} \ell \\
& =\frac{1}{\mathfrak{B}(b, c-b)} B_{\sigma, \delta ; \kappa}^{\mathrm{MC}}\left(b, c^{\prime}-a^{\prime}-b\right) .
\end{aligned}
$$

Theorem 7. If $\mathfrak{R e}\left(c^{\prime}\right)>\mathfrak{R} \mathbf{e}(b)>0$, then new generalization of confluent hypergeometric function $\varphi_{\sigma, \delta ; \kappa}^{M C}(b ; c ; \vartheta)$ is defined as follows:

$$
\begin{aligned}
& \varphi_{\sigma, \delta ; \kappa}^{\mathrm{MC}}(b ; c ; 9)=\frac{1}{\mathfrak{B}(b, c-b)} \int_{0}^{1} \ell^{\prime} \ell^{\prime-1}(1-\ell)^{c^{c} b-1} \exp (\vartheta \ell)_{1} F_{1}(\sigma ; \delta ; \kappa \ell(1-\ell)) \mathrm{d} \ell \\
& \varphi_{\sigma, \delta ; \kappa}^{\mathrm{MC}}(b ; c ; 9)=\frac{1}{\mathfrak{B}\left(b, c^{\prime}-b\right)} \int_{0}^{\infty} \varsigma^{{ }^{b-1}}(1+\varsigma)^{-{ }^{\prime}} \exp \left(\frac{\kappa \varsigma}{1+\varsigma}\right){ }_{1} F_{1}\left(\sigma ; \delta ; \frac{\kappa \varsigma}{(1+\varsigma)^{2}}\right) \mathrm{d} \varsigma, \\
& \varphi_{\sigma, \delta ; \kappa}^{\mathrm{MC}}(b ; c ; 9)=\frac{2}{\mathfrak{B}(b, c-b)} \int_{0}^{(\pi / 2)} \sin ^{2 b^{\prime}-1} \theta \cos ^{2 c^{\prime}-2 b-1} \theta \exp \left(\vartheta \sin ^{2} \theta\right){ }_{1} F_{1}\left(\sigma ; \delta ; \kappa \sin ^{2} \theta \cos ^{2} \theta\right) \mathrm{d} \theta .
\end{aligned}
$$

Proof: Considering the left hand side of (48), by using equation (39) and equation (8), we yield 


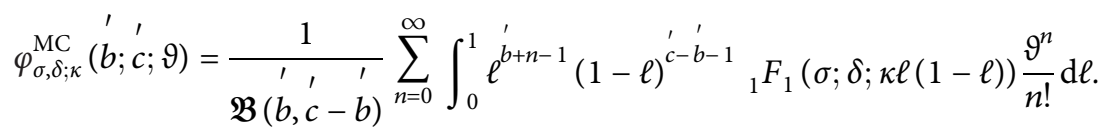

Changing the order of integration and summation, we obtain the required result $(48)$. By replacing $\ell=(\varsigma / 1+\varsigma)$ and $\ell=\sin ^{2} \theta$ separately in equation (48) and simplifying it, we obtain equations (49) and (50).

2.2.3. Differentiation Formulas on New Extension of Beta Function. To represent the new generalization of Gauss hypergeometric function and confluent hypergeometric function, we use the following conditions:

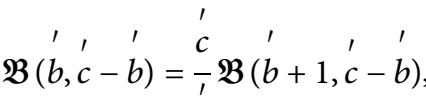

$$
\begin{aligned}
& \text { b } \\
& \stackrel{\prime}{(a)_{n+1}}=\stackrel{\prime}{a}(\stackrel{\prime}{a}+1)_{n} .
\end{aligned}
$$

Theorem 8. If $\mathfrak{R e}(a)>0, \mathfrak{R e}(c)>\Re \mathfrak{R}(b)>0$, then we have the following differential formula:

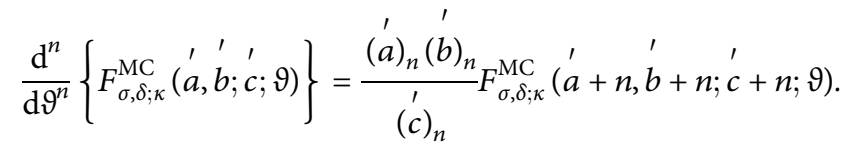

Proof: Taking the derivative of $F_{\sigma, \delta ; \kappa}^{\mathrm{MC}}(\dot{a}, \dot{b} ; \dot{c} ;$; 9$)$ with respect to 9 , we have

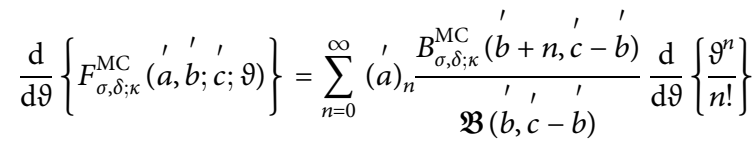

$$
\begin{aligned}
& =\sum_{n=1}^{\infty}(a)_{n}^{\prime} \frac{B_{\sigma, \delta ; \kappa}^{\mathrm{MC}}\left(b+n,{ }^{\prime} c-b\right)}{\mathfrak{B}^{\prime}{ }^{\prime}{ }^{\prime}} \frac{9^{n-1}}{(n-1) !}
\end{aligned}
$$

Replacing $n \longrightarrow n+1$ in the above equation (54), then it will reduce to

$$
\begin{aligned}
& \frac{\mathrm{d}}{\mathrm{d} \vartheta}\left\{F_{\sigma, \delta ; \kappa}^{\mathrm{MC}}(a, b ; c ; \vartheta)\right\}=\frac{{ }^{\prime} b}{\prime} \sum_{c}^{\infty}\left(\begin{array}{l}
\prime \\
c
\end{array}\right. \\
& =\frac{\stackrel{a}{a b}}{\frac{1}{c}} F_{\sigma, \delta ; \kappa}^{\mathrm{MC}}(\stackrel{\prime}{a+1, b+1 ; c+1 ; \vartheta) .}
\end{aligned}
$$

Recursive application of this technique provides us the general form of equation (53).

Theorem 9. For confluent hypergeometric function, we have the following differential formula:

$$
\frac{\mathrm{d}^{n}}{\mathrm{~d} \vartheta^{n}}\left\{\varphi_{\sigma, \delta ; \kappa}^{\mathrm{MC}}(b ; c ; \vartheta)\right\}=\frac{(b)_{n}}{{ }^{\prime}(c)_{n}} \varphi_{\sigma, \delta ; \kappa}^{\mathrm{MC}}(b+n ; c+n ; \vartheta) .
$$

Proof: Proof is same as in the above theorem.

2.2.4. Transformation Formula on New Extension of Beta Function

Theorem 10. For the new generalization of Gauss hypergeometric function with the condition $\left\{|\vartheta|<\pi ; \mathfrak{R e}\left({ }^{\prime}\right)\right.$ 
$>\mathfrak{R e}(b)>0\}$, we have the subsequent transformation formula:

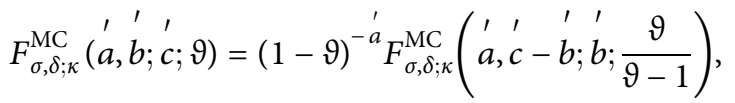

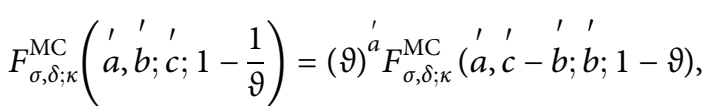

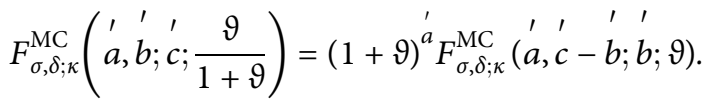

Proof: Using the expression,

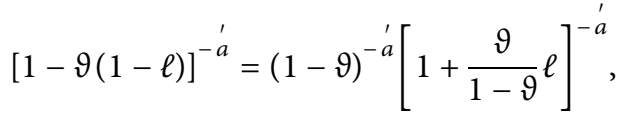

and replacing $\ell \longrightarrow 1-\ell$ in equation (41), we obtain

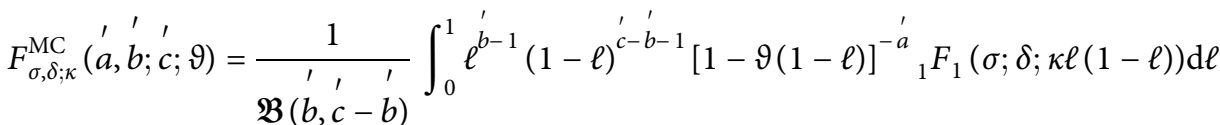

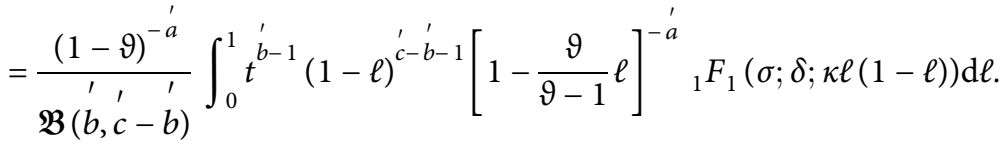

Hence,

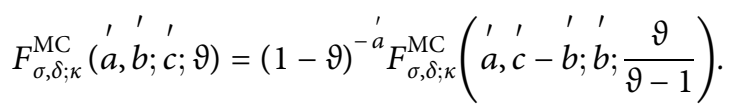

Replacing $\vartheta \longrightarrow 1-(1 / 9)$ in equation (57), we obtain the result given in equation (58), and again replacing 9 by $(\vartheta /(1+9))$ in equation (57), we get equation (59).

\section{New Extension of Riemann-Liouville (R-L) Fractional Derivative Operator}

In the past years, different kinds of extensions of R-L fractional operators were performed by many authors (see [19-22]). Here, we announce novel extension of R-L fractional derivative operator using new type of extended beta function and this function is also reducible into classical Riemann-Liouville fractional derivative operator with certain conditions.

The classical Riemann fractional derivative of $f(\vartheta)$ of order $\xi$ defined demarcated by

$$
D_{\vartheta}^{\xi} f(\vartheta)=\frac{1}{\Gamma(-\xi)} \int_{0}^{\vartheta}(\vartheta-\ell)^{-\xi-1} f(\ell) \mathrm{d} \ell, \quad\{\Re \mathfrak{R}(\xi)>0\},
$$

where the path of integration is a line from 0 to $\vartheta$ in the complex $\ell$ - plane when $\mathfrak{R e}(\xi) \geq 0$.

Let $n \in N$ be the smallest integer greater than $\mathfrak{R e}(\xi)$ and $n-1 \leq \mathfrak{R e}(\xi)<n$. Then,

$$
D_{\vartheta}^{\xi, n} f(\vartheta)=\frac{\mathrm{d}^{n}}{\mathrm{~d} \vartheta^{n}}\left\{D_{\vartheta}^{\xi-n} f(\vartheta)\right\}=\frac{\mathrm{d}^{n}}{\mathrm{~d} \vartheta^{n}}\left\{\frac{1}{\Gamma(n-\xi)} \int_{0}^{\vartheta}(\vartheta-\ell)^{n-\xi-1} f(\ell) \mathrm{d} \ell\right\}
$$

Here, we defined new definition of extended Riemann-Liouville (R-L) fractional derivative of $f(\vartheta)$ of order $\xi$ which is given $\mathrm{b}$

y

$$
\left.D_{\sigma, \delta ; \kappa}^{\mathrm{MC} ; \xi} f(\vartheta)=\frac{1}{\Gamma(-\xi)} \int_{0}^{\vartheta}(\vartheta-\ell)^{-\xi-1} f(\ell)\right){ }_{1} F_{1}\left(\sigma ; \delta ; \frac{\kappa \ell(\vartheta-\ell)}{\vartheta^{2}}\right) \mathrm{d} \ell
$$

where $\quad\left\{\mathfrak{R e}(\sigma)>0, \mathfrak{R e}(\delta)>0, \mathfrak{R e}(\xi)>0 ;|\kappa|<K ; K \in \mathfrak{R}^{+}\right.$ and less than 2.0335\} and for $n-1 \leq \mathfrak{R e}(\xi)<n, n \in N,|\kappa|<K ; K \in \mathfrak{R}^{+}$and less than 2.0335, $\mathfrak{R e}(\sigma)>0, \mathfrak{R e}(\delta)>0$, then 


$$
D_{\sigma, \delta ; \kappa}^{\mathrm{MC} ; \xi} f(\vartheta)=\frac{\mathrm{d}^{n}}{\mathrm{~d} \vartheta^{n}}\left\{\frac{1}{\Gamma(n-\xi)} \int_{0}^{\vartheta}(\vartheta-\ell)^{n-\xi-1} f(\ell){ }_{1} F_{1}\left(\sigma ; \delta ; \frac{\kappa \ell(\vartheta-\ell)}{\vartheta^{2}}\right) \mathrm{d} \ell\right\} .
$$

If we put $\kappa=0$ in the above equations (65) and (66), then it will become the classical Riemann-Liouville (R-L) fractional derivative equations (63) and (64).

Theorem 11. Let $n-1 \leq \Re e(\xi)<n$, for some $n \in N,|\kappa|<K$ and $\mathfrak{R e}(\xi)<\mathfrak{R e}(\mu)$. Then, we have

$$
D_{\sigma, \delta ; \kappa}^{\mathrm{MC} ;}\left\{\vartheta^{\mu}\right\}=\frac{B_{\sigma, \delta ; \kappa}^{\mathrm{MC}}(\mu+1,-\xi)}{\Gamma(-\xi)} \vartheta^{(\mu-\xi)} .
$$

Proof: We have from equation (65),

$$
D_{\sigma, \delta: \kappa}^{\mathrm{MC} ;}\left\{\vartheta^{\mu}\right\}=\frac{1}{\Gamma(-\xi)} \int_{0}^{\vartheta}(\vartheta-\ell)^{-\xi-1} \ell^{\mu}{ }_{1} F_{1}\left(\sigma ; \delta ; \frac{\kappa \ell(\vartheta-\ell)}{\vartheta^{2}}\right) \mathrm{d} \ell,
$$

and putting $\ell=\vartheta_{\varsigma}$ in the above equation, then we have

$$
D_{\sigma, \delta ; \kappa}^{\mathrm{MC} ; \xi}\left\{\vartheta^{\mu}\right\}=\frac{\vartheta^{-\xi+\mu}}{\Gamma(-\xi)} \int_{0}^{1}(1-\varsigma)^{-\xi-1} \varsigma^{\mu}{ }_{1} F_{1}(\sigma ; \delta ; \kappa \varsigma(1-\varsigma)) \mathrm{d} \varsigma=\frac{B_{\sigma, \delta: \kappa}^{\mathrm{MC}}(\mu+1,-\xi)}{\Gamma(-\xi)} \vartheta^{(\mu-\xi)}
$$

Theorem 12. Let $n-1 \leq \mathfrak{R e}(\mu-\xi)<n<\Re \mathfrak{R}(\mu), \quad n \in N$, $|\vartheta|<1, \quad \tau \in C,|\kappa|<K ; K \in \Re^{+}$and less than 2.0335, $\mathfrak{R e}(\sigma)>0, \mathfrak{R e}(\delta)>0$, then

$$
D_{\sigma, \delta ; \kappa}^{\mathrm{MC} ; \mu-\xi}\left\{\vartheta^{\mu-1}(1-\vartheta)^{-\tau}\right\}=\frac{\vartheta^{\xi-1}}{\Gamma(\xi-\mu)} \int_{0}^{1}(\varsigma)^{\mu-1}(1-\varsigma \vartheta)^{-\tau}(1-\varsigma)^{\xi-\mu-1}{ }_{1} F_{1}(\sigma ; \delta ; \kappa \varsigma(1-\varsigma)) \mathrm{d} \varsigma
$$

Proof: Taking the left hand side and using equation (65), we

have

$$
=\frac{1}{\Gamma(-\mu+\xi)} \int_{0}^{\vartheta}(\vartheta-\ell)^{-\mu+\xi-1} \ell^{\mu-1}(1-\ell)^{-\tau}{ }_{1} F_{1}\left(\sigma ; \delta ; \frac{\kappa \ell(\vartheta-\ell)}{\vartheta^{2}}\right) \mathrm{d} \ell
$$

and putting $\ell=\vartheta \varsigma$, then it becomes

$$
D_{\sigma, \delta ; \kappa}^{\mathrm{MC} ; \lambda-\xi}\left\{\vartheta^{\mu-1}(1-\vartheta)^{-\tau}\right\}=\frac{\vartheta^{\xi-1}}{\Gamma(\xi-\mu)} \int_{0}^{1}(\varsigma)^{\mu-1}(1-\varsigma \vartheta)^{-\tau}(1-\varsigma)^{\xi-\mu-1}{ }_{1} F_{1}(\sigma ; \delta ; \kappa \varsigma(1-\varsigma)) \mathrm{d} \varsigma
$$

3.1. Extended Riemann-Liouville (R-L) Derivative to a Function $f(\vartheta)$ Analytic at the Origin

$$
D_{\sigma, \delta ; \kappa}^{\mathrm{MC} ; \xi} f(\vartheta)=\sum_{n=0}^{\infty} \frac{1}{a_{n}} D_{\sigma, \delta ; \kappa}^{\mathrm{MC} ; \xi}\left\{\vartheta^{n}\right\}
$$

Theorem 13. Let function $f(\vartheta)$ be analytic at the origin with its power series given by $f(\vartheta)=\sum_{n=0}^{\infty} a_{n} \vartheta^{n},(|\vartheta|<\rho)$ for some $\rho \in R^{+}$, then
Proof: By the definition of extended Riemann-Liouville (RL) derivative operator and using $f(\vartheta)=\sum_{n=0}^{\infty} a_{n} \vartheta^{n}$ in it, we obtain

$$
D_{\sigma, \delta ; \kappa}^{\mathrm{MC} ; \xi} f(\vartheta)=\frac{1}{\Gamma(-\xi)} \int_{0}^{\vartheta}(\vartheta-\ell)^{-\xi-1} \sum_{n=0}^{\infty}\left({ }^{\prime}()_{n} \ell^{n}{ }_{1} F_{1}\left(\sigma ; \delta ; \frac{\kappa \ell(\vartheta-\ell)}{\vartheta^{2}}\right) \mathrm{d} \ell .\right.
$$



have

Changing the order of summation and integration, we

$$
\begin{aligned}
& =\sum_{n=0}^{\infty}(\stackrel{\prime}{a})_{n}\left\{\frac{1}{\Gamma(-\xi)} \int_{0}^{\vartheta}(\vartheta-\ell)^{-\xi-1} \ell^{n}{ }_{1} F_{1}\left(\sigma ; \delta ; \frac{\kappa \ell(\vartheta-\ell)}{\vartheta^{2}}\right) \mathrm{d} \ell\right\} \\
& =\sum_{n=0}^{\infty}(\stackrel{\prime}{a})_{n} D_{\sigma, \delta ; \kappa}^{\mathrm{MC} \xi \xi}\left\{\vartheta^{n}\right\} .
\end{aligned}
$$

Theorem 14. Let function $f(\vartheta)$ be analytic, at the origin with its power series expansion $f(\vartheta)=\sum_{n=0}^{\infty} a_{n} \vartheta^{n},(|\vartheta|<\rho)$ for some $\rho \in R^{+}$. Then, we have

$$
D_{\sigma, \delta ; \kappa}^{\mathrm{MC} ; \xi}\left\{\vartheta^{\mu-1} f(\vartheta)\right\}=\frac{\vartheta^{\mu-\xi-1}}{\Gamma(-\xi)} \sum_{n=0}^{\infty}\left({ }^{\prime} a\right)_{n} \vartheta^{n} \int_{0}^{1} \varsigma^{\mu+n-1}(1-\varsigma)^{-\xi-1}{ }_{1} F_{1}(\sigma ; \delta ; \kappa \varsigma(1-\varsigma)) \mathrm{d} \varsigma
$$

Proof. By using equation (73), in L.H.S. of the above equation, we have

$$
\begin{aligned}
D_{\sigma, \delta ; \kappa}^{\mathrm{MC} ; \xi}\left\{\vartheta^{\mu-1} f(\vartheta)\right\} & =\sum_{n=0}^{\infty}(\stackrel{a}{a})_{n} D_{\sigma, \delta ; \kappa}^{\mathrm{MC} ; \xi}\left\{\vartheta^{\mu-1} \vartheta^{n}\right\} \\
& =\sum_{n=0}^{\infty}(\stackrel{\prime}{a})_{n} \frac{1}{\Gamma(-\xi)} \int_{0}^{\vartheta}(\vartheta-\ell)^{-\xi-1} \ell^{\mu+n-1}{ }_{1} F_{1}\left(\sigma ; \delta ; \frac{\kappa \ell(\vartheta-\ell)}{\vartheta^{2}}\right) \mathrm{d} \ell
\end{aligned}
$$

and putting $\ell=\vartheta \varsigma$, then

$$
=\frac{1}{\Gamma(-\xi)} \int_{0}^{1} \vartheta^{-\xi+\mu-1}(1-\varsigma)^{-\xi-1} \varsigma^{\mu-1}{ }_{1} F_{1}(\sigma ; \delta ; \kappa \varsigma(1-\varsigma)) \sum_{n=0}^{\infty} a_{n}^{\prime}(\vartheta \varsigma)^{n} \mathrm{~d} \varsigma .
$$

The series given in equation (78), $\sum_{n=0}^{\infty} a^{\prime}{ }_{n}(\vartheta \varsigma)^{n}$, is uniformly convergent in disc $|\vartheta|<\rho$ for $0 \leq \varsigma \leq 1$ and the integral $\int_{0}^{1}\left|\varsigma^{\mu-1}(1-\varsigma)^{-\xi-1}{ }_{1} F_{1}(\sigma ; \delta ; \kappa \varsigma(1-\varsigma))\right| \mathrm{d} \varsigma$ is convergent provided that $\mathfrak{R e}(\mu)>0, \mathfrak{R e}(\xi)<0$ and $\kappa \in C,|\kappa|<K ; K \in R^{+}$and less than 2.033 5. Now, interchanging the order of summation and integration, we get the result given in equation (76).

\section{Data Availability}

No data were used to support this study.

\section{Conflicts of Interest}

The authors declare no conflicts of interest regarding the publication of this article.

\section{References}

[1] P. Agarwal, J. Choi, and R. B. Paris, "Extended RiemannLiouville fractional derivative operator and its applications," The Journal of Nonlinear Science and Applications, vol. 8, no. 5, pp. 451-466, 2015.
[2] M. A. Chaudhary and S. M. Zubair, "Generalized incomplete gamma function with applications," Journal of Computational and Applied Mathematics, vol. 55, no. 1, pp. 99-123, 1994.

[3] M. A. Chaudhary, A. Qadir, H. M. Srivastava, and R. B. Paris, "Extended hypergeometric and confluent hypergeometric function," Applied Mathematics and Computation, vol. 159, no. 2, pp. 589-602, 2004.

[4] J. Choi, M. I. Qureshi, A. H. Bhat, and J. Majid, "Reduction formulas for generalized hypergeometric series associated with new sequences and applications," Fractal Fract, vol. 5, p. 150, 2021.

[5] J. Choi, "Certain applications of generalized Kummer's summation formulas for ${ }^{2} \mathrm{~F}_{1}$," Symmetry, vol. 13, p. 1538, 2021.

[6] H. M. Srivastava and J. Choi, Zeta and Q-Zeta Functions and Associated Series and Integrals, Elsevier Science Publishers, New York, NY, USA, 2012.

[7] H. M. Srivastava and P. W. Karlsson, Multiple Gaussian Hypergeometric Series, Halsted Press, New York, NY, USA, 1985.

[8] J. Choi, A. K. Rathie, and R. K. Parmar, "Extension of Extended beta, hypergeometric and confluent hypergeometric functions," Honam Mathematical Journal, vol. 36, no. 2, pp. 339-367, 2014. 
[9] A. Erdelyi, W. Magnus, F. Oberhettinger, and F. G. Tricomi, Higher Transcendental Functions, Vol. I, McGraw-Hill Book Company, New York, NY, USA, 1953.

[10] N. Khan, T. Usman, and M. Aman, "Extended beta, hypergeometric confluent functions," 2019, https://arxiv.org/abs/ 1901.07397.

[11] A. A. Kilbas and N. Sebastian, "Generalized fractional integration of Bessel function of the first kind," Integral Transforms and Special Functions, vol. 19, no. 12, pp. 869-883, 2008.

[12] S. Mubeen, G. Rahman, K. S. Nisar, J. Choi, and M. Arshad, "An extended beta function and its properties," Far East Journal of Mathematical Sciences, vol. 102, no. 2, pp. 15451557, 2017.

[13] E. Özergin, M. A. Özarslan, and A. Altın, "Extension of gamma, beta and hypergeometric functions," Journal of Computational and Applied Mathematics, vol. 235, no. 16, pp. 4601-4610, 2011.

[14] R. K. Parmar, "A new generalization of gamma, beta, hypergeometric function," Le matematiche, vol. 68 , no. 2 , pp. 33-52, 2013.

[15] E. D. Rainville, Special functions, Macmillan Company, NewYork, NY, USA, 1971.

[16] M. Shadab, S. Jabee, and J. Choi, "An extended beta function and its applications," Far East Journal of Mathematical Sciences (FJMS), vol. 103, no. 1, pp. 235-251, 2018.

[17] M. A. Chaudhary, A. Qadir, M. Rafique, and S. M. Zubair, "Extension of Euler's beta function," Journal of Computational and Applied Mathematics, vol. 78, no. 1, pp. 19-32, 1997.

[18] M. Chand, H. Hachimi, and R. Rani, "New extension of beta function and its application," International Journal of Mathematics and Mathematical Sciences, vol. 2018, Article ID 6451592, 25 pages, 2018.

[19] M. Chand, J. C. Prajapati, J. Prajapati, E. Bonyah, and J. Kumar Bansal, "Fractional calculus and applications of family of extended generalized Gauss hypergeometric functions," Discrete \& Continuous Dynamical Systems-S, vol. 13, no. 3, pp. 539-560, 2020.

[20] I. S. Gradshteyn and I. M. Ryzhik, Table of Integrals, Series, and Products, Academia Press, Cambridge, MA, USA, Seventh edition, 2014.

[21] R. K. Parmar, P. Chopra, and R. B. Paris, "On an extension of extended beta and hypergeometric functions," 2015, https:// arxiv.org/abs/1502.06200.

[22] G. Rahman, K. S. Nisar, and Z. Tomovski, "A new extended of the Riemann-Liouville fractional derivative operator," 2018, https://arxiv.org/pdf/1801.05001. 